\begin{tabular}{|c|l|}
\hline Title & Palladium-Catalyzed Cross Coupling Reaction of Heteroary Itriol borates with A ryl Halides for Synthesis of Biaryls \\
\hline Author(s) & Y amamoto, Y asunori; Takizawa, Miho; Yu, Xiao-Qiang; Miy aura, Norio \\
\hline Citation & $\begin{array}{l}\text { Heterocycles, 80(1),359-368 } \\
\text { https://doi.org/10.3987/COM-09-S(S)30 }\end{array}$ \\
\hline Issue Date & $2010-01-01$ \\
\hline Doc URL & http://hdl.handle.net/2115/45016 \\
\hline Type & article (author version) \\
\hline File Information & Het80-1_359-368.pdf \\
\hline
\end{tabular}

Instructions for use 


\title{
PALLADIUM-CATALYZED CROSS-COUPLING REACTION OF HETEROARYLTRIOLBORATES WITH ARYL HALIDES FOR SYNTHESIS OF BIARYLS
}

\author{
Yasunori Yamamoto,* Miho Takizawa, Xiao-Qiang Yu, and Norio \\ Miyaura*
}

Graduate School of Engineering, Hokkaido University, Sapporo, 060-8628, Japan. E-mail: yasuyama@eng.hokudai.ac.jp or miyaura@eng.hokudai.ac.jp

\begin{abstract}
Cyclic triolborates possessing a heteroaromatic ring on the boron atom $\left[\mathrm{ArB}\left(\mathrm{OCH}_{2}\right)_{3} \mathrm{CHCH}_{3}\right] \mathrm{M}(\mathrm{M}=\mathrm{K}, \mathrm{Na}, \mathrm{Li})$ were prepared in high yields from heteroarylboronic acids, 1,1,1-tris(hydroxymethyl)ethane (triol) and $\mathrm{KOH}$ or $\mathrm{NaH}$. The corresponding lithium salts were synthesized from aryllithiums, $\mathrm{B}(\mathrm{OMe})_{3}$ and triol. They were air-stable white solids that were convenient for handling in air. High performance of these triolborates for metal-catalyzed bond-forming reactions was demonstrated in palladium-catalyzed cross-coupling reactions with haloarenes. Although the use of heteroarylboronic acids often results in very low yields due to competitive hydrolytic B-C bond cleavage with water under typical conditions using aqueous bases, triolborates possessing a 2-pyridyl, 3-pyridyl or 2-thiophenyl ring afforded biaryls in high yields at 50-120 ${ }^{\circ} \mathrm{C}$ in anhydrous DMF. There was a strong accelerating effect of $\mathrm{CuI}$ for reactions of 2- and 3-pyridylborate derivatives, whereas 2-thiophenyl derivatives reacted smoothly resulting in high yields in the absence of $\mathrm{CuI}$.
\end{abstract}

\section{INTRODUCTION}

Heteroaromatic biaryls are an important class of compounds due to the frequent occurrence of these fragments in natural products, pharmaceuticals, agrochemicals, and functional organic materials. ${ }^{1}$

\footnotetext{
ॠThis paper is dedicated to Prof. Akira Suzuki on the occasion of his 80th birthday.
} 
Cross-coupling reaction between arylmetal compounds and aryl electrophiles is a recent variant of traditional Ullman coupling for the synthesis of such biaryls. Although this protocol, which has been extensively studied in the past three decades, is now accessible with a variety of organometallic reagents and electrophiles, ${ }^{2}$ interest has recently been shown in the use of nonmetallic boron compounds because of their high stability in air and water and compatibility with a broad range of functional groups. The covalent $\mathrm{C}-\mathrm{B}$ bond of organoboronic acids is generally inert to transmetalation to transition metal complexes. Thus, nucleophilic tetracoordinated anionic complexes have been used successfully for metal-catalyzed bond-forming reactions of organoboron compounds. ${ }^{2,3}$ There are attempts at synthesizing stable ate-complexes such as $\left[\mathrm{RBF}_{3}\right] \mathrm{M} \quad\left(\mathrm{M}=\mathrm{K}, \quad \mathrm{NR}_{4}\right),{ }^{4} \quad\left[\mathrm{RB}(\mathrm{OH})_{3}\right] \mathrm{Na},{ }^{5} \quad \mathrm{ArB}(\mathrm{OR})_{3} \mathrm{Li}^{6}{ }^{6}$ $\left[\mathrm{RC} \equiv \mathrm{CBR}_{2}{ }_{2}(\mathrm{OR})\right] \mathrm{Li},{ }^{7,8}$ and $\left[\mathrm{Ar}_{4} \mathrm{~B}\right] \mathrm{Na}^{9}$ to overcome the limitations of in situ preparation of such ate-complexes from organoboranes and aqueous bases. Recently, we have developed ate-complexes, alkali metal cyclic triolborates that have exceptionally high levels of stability in air and water and reasonable solubility in organic solvents. High performance for bond-forming reactions was demonstrated in palladium-catalyzed cross-coupling, ${ }^{10}$ copper-catalyzed $N$-arylation ${ }^{11}$ and rhodium-catalyzed asymmetric 1,4-addition. ${ }^{12}$ Cross-couplings of heteroaromatic rings result in high yields in reactions between typical arylboronic acids and haloheteroarenes, but a reverse combination between heteroarylboronic acids and haloarenes often fails to give biaryls. This is mainly due to high sensitively of the B-C bond of electron-deficient heteroaryl rings to hydrolytic B-C bond cleavage with water, ${ }^{13}$ especially the B-C bonds adjacent to a nitrogen atom such as 2-pyridylboronic acid undergo very rapid cleavage. A recent advance in this field is the use of pinacol esters or diethanolamine esters of heteroarylboronic acids, which are more insensitive to water than are those of boronic acids. ${ }^{14} \mathrm{We}$ describe herein cross-coupling reaction of heteroaryltriolborates with haloarenes for the synthesis of biaryls (Scheme 1). Biaryls possessing a heteroaryl ring were obtained in high yields when the reaction was conducted in anhydrous DMF.

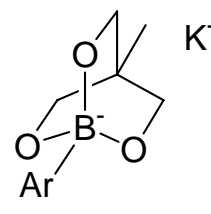

1

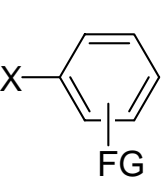

2

(1)

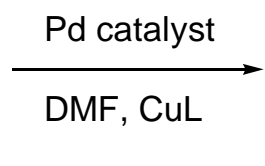

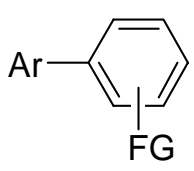

3

Ar= 2-thiophenyl, 2-pyridyl, 3-pyridyl 


\section{RESULTS AND DISCUSSION}

Synthesis of heteroaryltriolborates: We have reported two methods for the synthesis of cyclic triolborates. $^{10}$ Azeotropic removal of water upon treatment of 3-pyridylboronic acid (4) or 2-benzothiophenylboronic acid (6) with 1,1,1-tris(hydroxymethyl)ethane (triol) gave an ester intermediate (5 or 7). They were easily converted into triolborate (1a or 1b) by treatment with an alkali metal hydroxide or metal hydride (Scheme 2).

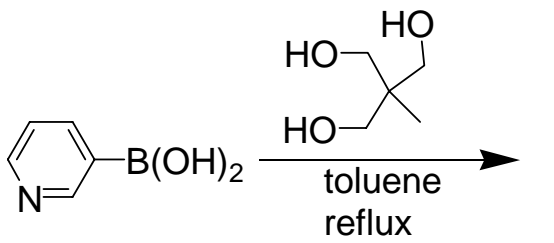

4<smiles>OCC(CO)(CO)CCCOc1cc2ccccc2s1</smiles>

6<smiles>CC1(C)COB(c2cccnc2)OC1</smiles>

$5(49 \%)$<smiles>CC1(CO)COB(c2cc3ccccc3s2)OC1CN</smiles>
$7(93 \%)$

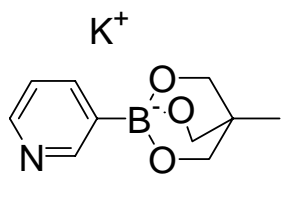

$1 \mathrm{a}(56 \%)$<smiles>CC12COB(OC1c1cc3ccccc3s1)O2</smiles>

1b $(78 \%)$

Scheme 2. Synthesis of Potassium and Sodium Heteroaryltriolborates

The corresponding lithium salts (1c-f) were synthesized by alkylation of $\mathrm{B}\left(\mathrm{O}^{i} \mathrm{Pr}\right)_{3}$ with aryllithiums, followed by ester exchange with triol (Scheme 3). By removing i-PrOH in vacuo from this mixture, arylboronates (1c-f) were obtained in high yields as air-stable white solids. This protocol afforded high yields for 2-pyridylboronates sensitive to B-C bond cleavage with water (1e and 1f).

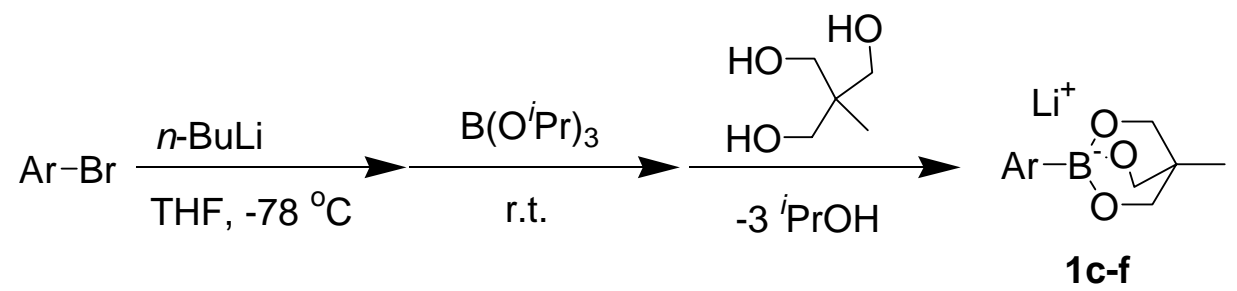<smiles>CC12CO[B-](c3cccs3)(OC1)OC2</smiles>

1c (99\%)<smiles></smiles>

1d (99\%)<smiles>CC12CO[B-](c3ccccn3)(OC1)OC2</smiles>

1 e $(86 \%)$<smiles>Cc1cccc([B-]23OCC(C)(CO2)CO3)n1</smiles>

1f $(96 \%)$

Scheme 3. Synthesis of Lithium Heteroaryltriolborates 
Scope and limitation: Cross-coupling reaction of potassium, sodium or lithium triolborates (2 eq) with aryl halides in anhydrous DMF gave biaryls in high yields (Table 1).

Table 1. Synthesis of Biaryls via Cross-Coupling of Heteroaryltriolborates (1) ${ }^{a}$

\begin{tabular}{|c|c|c|c|c|c|c|}
\hline entry & borate & Ar-X & $\begin{array}{l}\text { ligand } \\
(\mathrm{mol} \%)\end{array}$ & $\begin{array}{l}\mathrm{CuI} \\
\text { (eq.) }\end{array}$ & temp $/{ }^{\circ} \mathrm{C}$ & $\begin{array}{l}\text { yield } / \% b \\
\text { (compd No) }\end{array}$ \\
\hline 1 & 1a & $4-\mathrm{CH}_{3} \mathrm{COC}_{6} \mathrm{H}_{4} \mathrm{Br}$ & $\mathrm{PPh}_{3}(6.6)$ & 0.4 & 80 & $89(\mathbf{8 a})$ \\
\hline 2 & $1 \mathbf{b}$ & $4-\mathrm{CH}_{3} \mathrm{COC}_{6} \mathrm{H}_{4} \mathrm{Br}$ & XantPhos $^{c}$ (3.3) & non & 50 & $99(\mathbf{8 b})$ \\
\hline 3 & 1c & $4-\mathrm{CH}_{3} \mathrm{COC}_{6} \mathrm{H}_{4} \mathrm{Br}$ & $\operatorname{JohnPhos}^{d}(6.6)$ & non & 50 & $96(\mathbf{8 c})$ \\
\hline 4 & 1d & $4-\mathrm{MeOC}_{6} \mathrm{H}_{4} \mathrm{I}$ & JohnPhos $^{d}(13.2)$ & non & 50 & $74^{e}(\mathbf{8 d})$ \\
\hline 5 & 1e & $4-\mathrm{O}_{2} \mathrm{NC}_{6} \mathrm{H}_{4} \mathrm{Br}$ & $\mathrm{PPh}_{3}(6.6)$ & 0.2 & 80 & $90(\mathbf{8 e})$ \\
\hline 6 & 1e & $4-\mathrm{O}_{2} \mathrm{NC}_{6} \mathrm{H}_{4} \mathrm{Br}$ & $\mathrm{PPh}_{3}(6.6)$ & 0.1 & 80 & $76(8 \mathbf{e})$ \\
\hline 7 & 1e & $4-\mathrm{O}_{2} \mathrm{NC}_{6} \mathrm{H}_{4} \mathrm{Br}$ & $\mathrm{PPh}_{3}(6.6)$ & non & 80 & $47(\mathbf{8 e})$ \\
\hline 8 & 1e & 4- $\mathrm{CH}_{3} \mathrm{COC}_{6} \mathrm{H}_{4} \mathrm{Br}$ & $\mathrm{PPh}_{3}(6.6)$ & 0.2 & 80 & 75 (8f) \\
\hline 9 & 1e & 2-Bromopyridine & $\mathrm{PPh}_{3}(6.6)$ & 0.4 & 80 & $70(\mathbf{8 g})$ \\
\hline 10 & 1e & $4-\mathrm{MeC}_{6} \mathrm{H}_{4} \mathrm{I}$ & XantPhos $^{c}(6.6)$ & 0.4 & 100 & $77^{e}(\mathbf{8 h})$ \\
\hline 11 & 1e & $4-\mathrm{MeOC}_{6} \mathrm{H}_{4} \mathrm{I}$ & XantPhos $^{c}$ (6.6) & 0.4 & 100 & $74^{e}(\mathbf{8 i})$ \\
\hline 12 & 1f & $4-\mathrm{O}_{2} \mathrm{NC}_{6} \mathrm{H}_{4} \mathrm{I}$ & JohnPhos $^{d}$ (13.2) & 1.2 & 120 & $77^{e, f}(\mathbf{8 j})$ \\
\hline
\end{tabular}

${ }^{a} \mathrm{~A}$ mixture of haloarene $(0.5 \mathrm{mmol})$, triolborate $(\mathbf{1}, 1.0 \mathrm{mmol}), \mathrm{Pd}(\mathrm{OAc})_{2}(3 \mathrm{~mol} \%)$, ligand $(\mathrm{Pd}: \mathrm{P}=1: 2.2)$ and $\mathrm{CuI}$ (if used, 0.2 to $1.2 \mathrm{eq}$ ) in anhydrous DMF was stirred for $22 \mathrm{~h} .{ }^{b}$ Isolated yields by chromatography.

${ }^{c}$ XantPhos = 9,9-Dimethyl-4,5-bis(diphenylphosphino)xanthene. ${ }^{d}$ JohnPhos $=2$-(Di-t-butylphosphino)biphenyl.

${ }^{e} 6 \mathrm{~mol} \%$ of $\mathrm{Pd}(\mathrm{OAc})_{2}$ was used. ${ }^{f} 2.5$ eq of triolborate was used.

The presence of CuI was effective for increasing the coupling yields of 3-pyridyl- (1a), 2-pyridyl- (1e) and 6-methyl-2-pyridylborate (1f) (entries 1, 5-12), whereas it had no effect for more electron-rich 6-methoxy-3-pyridylborate (1d) (entry 4). The reaction of $\mathbf{1 d}$ proceeded smoothly at $50{ }^{\circ} \mathrm{C}$ in the absence of CuI, but 1a, 1e and $1 \mathbf{f}$ required much higher temperatures $\left(80-100{ }^{\circ} \mathrm{C}\right)$ in the presence of CuI. Thus, the results suggested that $\mathrm{CuI}$ requirement and temperature mainly depend on nucleophilicity of the pyridine rings. There has not yet been a mechanistic study; however, the rate of transmetalation ${ }^{2 f}$ and reductive 
elimination $^{15}$, among the three processes involved in the catalytic cycle, can be slowed down by decreasing the electron density on aromatic rings. The yields were generally increased by increasing the amount of $\mathrm{CuI}$ (entries 5-7). On the other hand, both 2-thiophenyl- (1c) and 2-benzothiophenylborate (1b) reacted smoothly at $50{ }^{\circ} \mathrm{C}$ in the absence of $\mathrm{CuI}$ (entries 2 and 3). Thus, electron-deficient 2- and 3-pyridylborate derivatives required $\mathrm{CuI}$ as a co-catalyst. The role of $\mathrm{CuI}$ is not known; however, such an effect of copper salts has been successfully utilized in analogous coupling reactions of heteroarylboron compounds. ${ }^{14 \mathrm{~d}-\mathrm{g}}$ In a preliminary study on the effects of catalysts, coupling between 4-tolyltriolborate (1, $\mathrm{Ar}=4$-methylphenyl) and 4-trifluorobromobenzene at room temperature in aqueous DMF showed the following ligand effects: dppf $(63 \%)<(t \text {-Bu })_{3} \mathrm{P}(70 \%)<\mathrm{Ph}_{3} \mathrm{P}(81 \%)<$ XantPhos $(90 \%)<$ JohnPhos (95\%). Although the best ligand for each combination of two aryl rings did not show clear correlation with chemical property of triolborates or haloarenes for oxidative addition or transmetalation, the best yield was obtained when $\mathrm{Ph}_{3} \mathrm{P}$, XantPhos or JohnPhos was used for $\mathrm{Pd}(\mathrm{OAc})_{2}$. Under these reaction conditions, biaryls possessing a heteroaryl ring were synthesized in high yields. Bromides were used at 80 ${ }^{\circ} \mathrm{C}$ for substrates possessing an electron-withdrawing group (entries 1 and 5-9), but electron-rich 4-tolyl and 4-methoxyphenyl derivatives reacted very slowly at $80{ }^{\circ} \mathrm{C}$. Thus, iodides were used at $100{ }^{\circ} \mathrm{C}$ for these two substrates (entries 10 and 11). 6-Methyl-2-pyridylborate (1f) had strong resistance for an unknown reason (entry 12), though unsubstituted 2-pyridylborate (1e) smoothly coupled with representative halides (entries 5-11).

In conclusion, our triolborates gave a simple access to heterobiaryls via palladium-catalyzed cross-coupling reaction with haloarenes. Since such ate-complexes are key species for various ionic reactions of boron compounds, extension to other bond-forming reactions will be the topic of further accounts from this laboratory.

\section{EXPERIMENTAL}

General: All reactions were conducted under an atmosphere of nitrogen. Glassware was oven dried at $130^{\circ} \mathrm{C}$ and allowed to cool under a stream of dry nitrogen. DMF was distilled from $\mathrm{CaH}_{2}$ before use. All chemicals were purchased from Aldrich, Wako, TCI, or Kanto Chemicals and used as received.

Potassium 3-pyridylborate (1a): 3-Pyridylboronic acid (4: 38 mmol) and 1,1,1-tris(hydroxymethyl)ethane $(38 \mathrm{mmol})$ were dissolved in toluene $(90 \mathrm{~mL})$. Water was removed by azeotropic distillation by the Dean-Stark method for $4 \mathrm{~h}$. The resulting mixture was extracted with AcOEt, washed with brine, and dried over anhydrous $\mathrm{MgSO}_{4}$. The filtrate was concentrated in vacuo to give crude 5 (49\%). The crude 5 and $\mathrm{KOH}(13 \mathrm{mmol})$ were dissolved in toluene $(40 \mathrm{~mL})$ and heated at reflux for $4 \mathrm{~h}$ by the Dean-Stark method. The potassium triolborate (1a) that precipitated was collected by filtration, washed with THF, and dried under reduced pressure. 
${ }^{1} \mathrm{H}$ NMR (400 MHz, DMSO-d $\left._{6}\right): \delta 0.50$ (s, 3H), 3.59 (s, 3H), 6.98 (br s, 1H), 7.60 (br s, 1H), 8.15 (br s, 1H), 8.43 (br s, $1 \mathrm{H}) ;{ }^{13} \mathrm{C}$ NMR (100 MHz, DMSO-d $\left.d_{6}\right): \delta 16.4,34.7,73.7,122.0,139.5,145.7,153.6$. MS (FAB') m/z (\%): 206 ([M-K]', 8), 205 (2), 192 (4), 186 (3), 178 (4), 175 (6), 153 (7), 149 (7), 148 (100), 146 (18); HRMS (FAB $\left.{ }^{-}\right): \mathrm{m} / \mathrm{z}$ calcd. for $\mathrm{C}_{10} \mathrm{H}_{13} \mathrm{BNO}_{3} 206.0989$, found 206.1000.

Sodium 2-benzo[b]thiophenylborate (1b): 2-benzo[b]thiophenyl boronic acid (6: $100 \mathrm{mmol}$ ) and 1,1,1-tris(hydroxymethyl)ethane $(100 \mathrm{mmol})$ were dissolved in toluene $(200 \mathrm{~mL})$. Water was removed by azeotropic distillation by the Dean-Stark method for $4 \mathrm{~h}$. The resulting mixture was extracted with AcOEt, washed with brine, and dried over anhydrous $\mathrm{MgSO}_{4}$. The filtrate was concentrated in vacuo to give crude 7 (93\%). A solution of crude 7 in ether was added to a stirred solution of $\mathrm{NaH}$ (120 mmol) in ether at $0{ }^{\circ} \mathrm{C}$. The resulting mixture was stirred for $0.5 \mathrm{~h}$ and then allowed to warm to room temperature. The mixture was then stirred for $20 \mathrm{~h}$. The sodium triolborate (1b) that precipitated was collected by filtration, washed with ether, and dried under reduced pressure. ${ }^{1} \mathrm{H}$ NMR (400 MHz, DMSO- $\left.d_{6}\right): \delta 0.82(\mathrm{~s}, 3 \mathrm{H}), 3.67$ $(\mathrm{s}, 6 \mathrm{H}), 7.31-7.34(\mathrm{~m}, 2 \mathrm{H}), 7.70(\mathrm{~s}, 1 \mathrm{H}), 7.84-7.86(\mathrm{~m}, 1 \mathrm{H}), 7.92-7.94(\mathrm{~m}, 1 \mathrm{H}) ;{ }^{13} \mathrm{C} \mathrm{NMR}(100 \mathrm{MHz}$,

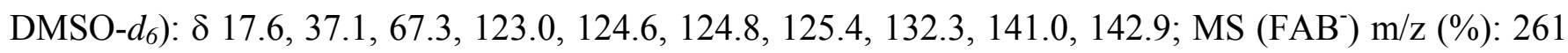
([M-Na]', 35), 168 (26), 122 (16); HRMS (FAB'): m/z calcd. for $\mathrm{C}_{13} \mathrm{H}_{14} \mathrm{BO}_{3} \mathrm{~S}$ 261.0762, found 261.0769.

Lithium 2-pyridylborate $(1 \mathbf{e})^{10}: n$-BuLi $(110 \mathrm{mmol})$ in hexane was added to a stirred solution of 2-bromopyridine $(100 \mathrm{mmol})$ in THF $(400 \mathrm{~mL})$ at $-78{ }^{\circ} \mathrm{C}$. The resulting mixture was stirred for $1 \mathrm{~h}$ at -78 ${ }^{\circ} \mathrm{C}$, and then a solution of triisopropylborate $(120 \mathrm{mmol})$ was added. The mixture was stirred for $2 \mathrm{~h}$ at $-78{ }^{\circ} \mathrm{C}$ and then allowed to warm to room temperature. A solution of 1,1,1-tris(hydroxymethyl)ethane (100 mmol) in THF $(150 \mathrm{~mL})$ was then added, and the resulting mixture was stirred for $5 \mathrm{~h}$. Concentration to dryness under reduced pressure gave 1e $(86 \%) .{ }^{1} \mathrm{H}$ NMR (400 MHz, DMSO- $\left.d_{6}\right): \delta 0.52$ (s, 3H), 3.67 (s, 3H), 7.05 (t, $J=5.56 \mathrm{~Hz}, 1 \mathrm{H}), 7.33$ (d, $J=7.13 \mathrm{~Hz}, 1 \mathrm{H}), 7.50$ (t, $J=7.26 \mathrm{~Hz}, 1 \mathrm{H}), 8.24$ $(\mathrm{d}, J=4.89 \mathrm{~Hz}, 1 \mathrm{H}) ;{ }^{13} \mathrm{C}$ NMR $\left(100 \mathrm{MHz}, \mathrm{DMSO}-\mathrm{d}_{6}\right): \delta 15.9,34.7,73.5,120.8,127.0,134.4,146.9 ;{ }^{11} \mathrm{~B}$ NMR (128 MHz, DMSO-d $_{\text {): }} \delta$ 1.65; MS (FAB'): m/z (\%): 206 ([M-Li]', 19), 205 (6), 185 (9), 154 (39), 152 (11), 149 (12), 148 (100), 146 (33); HRMS (FAB ${ }^{-}$: m/z calcd. for $\mathrm{C}_{10} \mathrm{H}_{13} \mathrm{BNO}_{3}$ 206.0989, found 206.0971 .

Lithium 2-thienylborate (1c): ${ }^{1} \mathrm{H}$ NMR (400 MHz, DMSO-d 6 ): $\delta 0.46$ (s, 3H), 3.54 (s, 3H), 6.74 (d, $J=$ $3.35 \mathrm{~Hz}, 1 \mathrm{H}), 6.81(\mathrm{t}, J=4.46 \mathrm{~Hz}, 1 \mathrm{H}), 7.07(\mathrm{~d}, J=4.46 \mathrm{~Hz}, 1 \mathrm{H}) ;{ }^{13} \mathrm{C}$ NMR $\left(100 \mathrm{MHz}, \mathrm{DMSO}-\mathrm{d}_{6}\right): \delta$ 16.4, 35.0, 73.8, 12.5, 126.2, 126.9; ${ }^{11} \mathrm{~B}$ NMR (128 MHz, DMSO-d 6 ): $\delta$ 0.85; MS (FAB $)$ m/z (\%): 211 ([M-Li]', 9), 192 (4), 175 (8), 154 (17), 153 (5), 149 (9), 148 (100), 146 (22); HRMS (FAB`): m/z calcd. for $\mathrm{C}_{9} \mathrm{H}_{12} \mathrm{BO}_{3} \mathrm{~S} 211.0600$, found 211.0585 .

Lithium 6-methoxy-3-pyridylborate (1d): ${ }^{1} \mathrm{H}$ NMR (400 MHz, DMSO- $\left.d_{6}\right): \delta 0.48$ (s, 3H), 3.56 (s, 3H), $3.74(\mathrm{~s}, 3 \mathrm{H}), 6.42(\mathrm{~d}, J=8.00 \mathrm{~Hz}, 1 \mathrm{H}), 7.52(\mathrm{~d}, J=7.59 \mathrm{~Hz}, 1 \mathrm{H}), 7.96(\mathrm{~s}, 1 \mathrm{H}) ;{ }^{13} \mathrm{C} \mathrm{NMR}(100 \mathrm{MHz}$, 
DMSO-d $\left.{ }_{6}\right): \delta 16.4,34.8,52.5,73.1,107.9,143.2,150.1,179.5 ;$ MS (FAB $\left.{ }^{-}\right)$m/z (\%): 236 ([M-Li]', 100), 235 (24); HRMS (FAB'): m/z calcd. for $\mathrm{C}_{11} \mathrm{H}_{15} \mathrm{BNO}_{4}$ 236.1094, found 236.1110.

Lithium 6-methyl-2-pyridylborate (1f): ${ }^{1} \mathrm{H}$ NMR (400 MHz, DMSO- $\left.d_{6}\right): \delta 0.54(\mathrm{~s}, 3 \mathrm{H}), 2.35$ (s, 3H), $3.67(\mathrm{~s}, 3 \mathrm{H}), 6.91(\mathrm{~d}, J=7.56 \mathrm{~Hz}, 1 \mathrm{H}), 7.12(\mathrm{~d}, J=7.56 \mathrm{~Hz}, 1 \mathrm{H}), 7.40(\mathrm{t}, J=7.56 \mathrm{~Hz}, 1 \mathrm{H}) ;{ }^{13} \mathrm{C} \mathrm{NMR}$ (100 MHz, DMSO-d $\left.d_{6}\right): \delta 15.9,23.8,34.6,73.5,119.9,124.0,134.6,154.7$.

Typical Procedures for Cross-Coupling Reaction: Heteroaryl cyclic triolborate (1.0 mmol), palladium acetate (3 or $6 \mathrm{~mol} \%)$, ligand $(\mathrm{Pd}: \mathrm{P}=1: 2.2)$ and $\mathrm{CuI}$ (if used, 0.2 to 1.2 equiv) in dry DMF $(3.0 \mathrm{~mL})$ were placed in a flask under nitrogen. Aryl halide $(0.5 \mathrm{mmol})$ was added to the reaction mixture, and then the mixture was stirred for $22 \mathrm{~h}$ at the temperature shown in Table 1 . The reaction was quenched with $\mathrm{NH}_{4} \mathrm{Cl}$ (sat. aq.). The resulting mixture was extracted with AcOEt, washed with brine, and dried over anhydrous $\mathrm{MgSO}_{4}$. The filtrate was concentrated in vacuo, and then chromatography over silica gel gave the desired product.

3-(4-Acetylphenyl)pyridine (8a) ${ }^{16}:{ }^{1} \mathrm{H} \mathrm{NMR}\left(400 \mathrm{MHz}, \mathrm{CDCl}_{3}\right): \delta 2.63$ (s, 3H), 7.39 (dd, $J=4.8,7.9 \mathrm{~Hz}$, $1 \mathrm{H}), 7.67(\mathrm{~d}, J=8.4 \mathrm{~Hz}, 2 \mathrm{H}), 7.90(\mathrm{dt}, J=1.9,7.9 \mathrm{~Hz}, 1 \mathrm{H}), 8.06(\mathrm{~d}, J=8.4 \mathrm{~Hz}, 2 \mathrm{H}), 8.64(\mathrm{dd}, J=1.5$, $4.8 \mathrm{~Hz}, 1 \mathrm{H}), 8.87(\mathrm{~d}, J=2.0 \mathrm{~Hz}, 1 \mathrm{H}) ;{ }^{13} \mathrm{C} \mathrm{NMR}\left(100 \mathrm{MHz}, \mathrm{CDCl}_{3}\right): \delta 26.4,123.4,12.9,128.8,134.2$, 135.0, 136.2, 142.0, 148.0, 149.0, 197.2; MS (EI) m/z (\%): $197\left(\mathrm{M}^{+}, 52\right), 182$ (100), 154 (35), 127 (23); HRMS (EI): m/z calcd. for $\mathrm{C}_{13} \mathrm{H}_{11} \mathrm{NO} 197.0841$, found 197.0833 .

2-(4-Acetylphenyl)thiophene (8b) ${ }^{17}:{ }^{1} \mathrm{H}$ NMR (400 MHz, $\left.\mathrm{CDCl}_{3}\right): \delta 2.62(\mathrm{~s}, 3 \mathrm{H}), 7.13(\mathrm{dd}, J=3.6,5.0$ $\mathrm{Hz}, 1 \mathrm{H}), 7.37$ (dd, $J=0.9,5.0 \mathrm{~Hz}, 1 \mathrm{H}), 7.44$ (dd, $J=0.9,3.6 \mathrm{~Hz}, 1 \mathrm{H}), 7.70$ (d, $J=1.8,8.6 \mathrm{~Hz}, 2 \mathrm{H}), 7.97$ $(\mathrm{dt}, J=1.8,8.6 \mathrm{~Hz}, 2 \mathrm{H}) ;{ }^{13} \mathrm{C} \mathrm{NMR}\left(100 \mathrm{MHz}, \mathrm{CDCl}_{3}\right): \delta 26.5,124.5,125.6,126.4,128.3,129.0,135.6$, 138.7, 142.8, 197.3; MS (EI) m/z (\%): $202\left(\mathrm{M}^{+}, 69\right), 187$ (100), 159 (20), 115 (35); HRMS (EI): m/z calcd. for $\mathrm{C}_{12} \mathrm{H}_{10} \mathrm{OS} 202.0452$, found 202.0452.

4-Acetylphenylbenzo[b]thiophen (8c) ${ }^{18}:{ }^{1} \mathrm{H}$ NMR $\left(400 \mathrm{MHz}, \mathrm{CDCl}_{3}\right): \delta 2.62$ (s, 3H), 7.36 (br s, 2H), 7.65 (s, 1H), 7.79 (br s, 4H), $8.00(\mathrm{~d}, J=6.3 \mathrm{~Hz}, 2 \mathrm{H}) ;{ }^{13} \mathrm{C} \mathrm{NMR}\left(100 \mathrm{MHz}, \mathrm{CDCl}_{3}\right): \delta 26.6,121.2,122.3$, 124.0, 124.8, 125.0, 126.3, 129.0, 136.4, 138.7, 139.9, 140.4, 197.2; MS (EI) m/z (\%): $252\left(\mathrm{M}^{+}, 81\right), 237$ (100), 209 (27), 208 (39), 165 (37), 139 (6), 118 (9), 104 (13); HRMS (EI): m/z calcd. for $\mathrm{C}_{16} \mathrm{H}_{12} \mathrm{OS}$ 252.0609 , found 252.0599.

2-Methoxy-5-(4-methoxyphenyl)pyridine (8d) ${ }^{19}:{ }^{1} \mathrm{H}$ NMR (400 MHz, $\left.\mathrm{CDCl}_{3}\right): \delta 3.82$ (s, 3H), 3.96 (s, 3H), $6.78(\mathrm{~d}, J=8.6 \mathrm{~Hz}, 1 \mathrm{H}), 6.96(\mathrm{~d}, J=8.8 \mathrm{~Hz}, 2 \mathrm{H}), 7.43(\mathrm{~d}, J=8.8 \mathrm{~Hz}, 2 \mathrm{H}), 7.72(\mathrm{dd}, J=2.5,8.5 \mathrm{~Hz}$, $1 \mathrm{H}), 8.34(\mathrm{~d}, J=2.4 \mathrm{~Hz}, 1 \mathrm{H}) ;{ }^{13} \mathrm{C} \mathrm{NMR}\left(100 \mathrm{MHz}, \mathrm{CDCl}_{3}\right): \delta 53.4,55.2,110.6,114.3,127.6,129.7$, 130.3, 137.0, 144.4, 159.1, 163.1; MS (EI) m/z (\%): $215\left(\mathrm{M}^{+}, 100\right), 200$ (22), 186 (30), 172 (13), 145 (9), 102 (10); HRMS (EI): m/z calcd. for $\mathrm{C}_{13} \mathrm{H}_{13} \mathrm{NO}_{2} 215.0946$, found 215.0946.

2-(4-Nitrophenyl)pyridine (8e) $)^{20}:{ }^{1} \mathrm{H}$ NMR (400 MHz, $\left.\mathrm{CDCl}_{3}\right): \delta 7.34(\mathrm{~m}, 1 \mathrm{H}), 7.81(\mathrm{~m}, 2 \mathrm{H}), 8.17(\mathrm{~d}, J$ $=8.9 \mathrm{~Hz}, 1 \mathrm{H}), 8.32(\mathrm{~d}, J=8.9 \mathrm{~Hz}, 1 \mathrm{H}), 8.75(\mathrm{~d}, J=4.8 \mathrm{~Hz}, 1 \mathrm{H}) ;{ }^{13} \mathrm{C} \mathrm{NMR}\left(100 \mathrm{MHz}, \mathrm{CDCl}_{3}\right): \delta 121.1$, 
123.4, 123.9, 127.5, 137.0, 145.1, 148.0, 150.0, 154.6; MS (EI) m/z (\%): $200\left(\mathrm{M}^{+}, 100\right), 170(23), 154$ (81), 142 (19), 127 (53); HRMS (EI): m/z calcd. for $\mathrm{C}_{11} \mathrm{H}_{8} \mathrm{~N}_{2} \mathrm{O}_{2} 200.0586$, found 200.0580.

2-(4-Acetylphenyl)pyridine (8f) $)^{21}:{ }^{1} \mathrm{H} \mathrm{NMR}\left(400 \mathrm{MHz}, \mathrm{CDCl}_{3}\right): \delta 2.63$ (s, 3H), 7.28 (q, $\left.J=4.5 \mathrm{~Hz}, 1 \mathrm{H}\right)$, 7.73-7.74 (m, 2H), 8.07 (q, $J=9.1 \mathrm{~Hz}, 4 \mathrm{H}), 8.72(\mathrm{dt}, J=1.4,4.5 \mathrm{~Hz}, 1 \mathrm{H}) ;{ }^{13} \mathrm{C} \mathrm{NMR}\left(100 \mathrm{MHz}, \mathrm{CDCl}_{3}\right)$ : $\delta 26.6,120.9,122.8,126.9,128.7,136.8,137.0,143.4,149.8,155.9,197.7$; MS (EI) m/z (\%): $197\left(\mathrm{M}^{+}\right.$, 53), 182 (100), 154 (44), 127 (12); HRMS (EI): m/z calcd. for $\mathrm{C}_{13} \mathrm{H}_{11} \mathrm{NO}$ 197.0841, found 197.0835.

2,2'-bipyridine (8g) ${ }^{22}:{ }^{1} \mathrm{H}$ NMR (400 MHz, $\left.\mathrm{CDCl}_{3}\right): \delta 7.30(\mathrm{~m}, 2 \mathrm{H}), 7.81$ (dt, J=1.5, $\left.7.8 \mathrm{~Hz}, 2 \mathrm{H}\right), 8.39$ $(\mathrm{d}, J=7.9 \mathrm{~Hz}, 2 \mathrm{H}), 8.68(\mathrm{~d}, J=4.0 \mathrm{~Hz}, 2 \mathrm{H}) ;{ }^{13} \mathrm{C} \mathrm{NMR}\left(100 \mathrm{MHz}, \mathrm{CDCl}_{3}\right): \delta 121.1,123.7,136.9,149.1$, 156.0; MS (EI) m/z (\%): $156\left(\mathrm{M}^{+}, 100\right), 128$ (19), 103 (3), 78 (16), 51 (10); HRMS (EI): m/z calcd. for $\mathrm{C}_{12} \mathrm{H}_{11} \mathrm{NO} 156.0687$, found 156.0682 .

2-(4-Methylphenyl)pyridine (8h) ${ }^{23}:{ }^{1} \mathrm{H}$ NMR (400 MHz, $\left.\mathrm{CDCl}_{3}\right): \delta 2.38(\mathrm{~s}, 3 \mathrm{H}), 7.15(\mathrm{~m}, 1 \mathrm{H}), 7.26(\mathrm{~d}$, $J=7.9 \mathrm{~Hz}, 2 \mathrm{H}), 7.67(\mathrm{~m}, 2 \mathrm{H}), 7.88(\mathrm{~d}, J=8.2 \mathrm{~Hz}, 1 \mathrm{H}), 8.66(\mathrm{dt}, J=1.3,4.7 \mathrm{~Hz}, 1 \mathrm{H}) ;{ }^{13} \mathrm{C} \mathrm{NMR}(100$ $\left.\mathrm{MHz}, \mathrm{CDCl}_{3}\right): \delta 21.2,120.2,121.7,126.7,129.4,136.6,138.9,149.5,157.4 ; \mathrm{MS}(\mathrm{EI}) \mathrm{m} / \mathrm{z}(\%): 169\left(\mathrm{M}^{+}\right.$, 100), 154 (7); HRMS (EI): calcd. for $\mathrm{C}_{12} \mathrm{H}_{11} \mathrm{~N}$ 169.0891, found 169.0881.

2-(4-Methoxyphenyl)pyridine (8i) ${ }^{24}:{ }^{1} \mathrm{H} \mathrm{NMR}\left(400 \mathrm{MHz}, \mathrm{CDCl}_{3}\right): \delta 3.77(\mathrm{~s}, 3 \mathrm{H}), 6.91(\mathrm{~d}, J=8.9 \mathrm{~Hz}$, 2H), 7.05-7.10 (m, 1H), 7.56-7.65 (m, 2H), $7.87(\mathrm{dt}, J=2.4,8.9 \mathrm{~Hz}, 2 \mathrm{H}), 8.56(\mathrm{~d}, J=4.7 \mathrm{~Hz}, 1 \mathrm{H}) ;{ }^{13} \mathrm{C}$ NMR (100 MHz, $\left.\mathrm{CDCl}_{3}\right): \delta 55.3,114.1,119.8,121.3,128.1,132.0,136.6,149.5,157.0,160.4$; MS (EI) m/z (\%): $185\left(\mathrm{M}^{+}, 100\right), 170$ (30), 142 (28); HRMS (EI): m/z calcd. for $\mathrm{C}_{12} \mathrm{H}_{11} \mathrm{NO} 185.0841$, found 185.0837 .

2-Methyl-6-(4-nitrophenyl)pyridine (8j): ${ }^{1} \mathrm{H} \mathrm{NMR}\left(400 \mathrm{MHz}, \mathrm{CDCl}_{3}\right): \delta 2.65$ (s, 3H), 7.20 (d, $J=7.5$, $1 \mathrm{H}), 7.60(\mathrm{~d}, J=7.7 \mathrm{~Hz}, 1 \mathrm{H}), 7.70(\mathrm{~d}, J=7.7 \mathrm{~Hz}, 1 \mathrm{H}), 8.31(\mathrm{~d}, J=8.9 \mathrm{~Hz}, 2 \mathrm{H}), 8.17$ (d, $J=8.8 \mathrm{~Hz}, 2 \mathrm{H})$; ${ }^{13} \mathrm{C}$ NMR (100 MHz, $\left.\mathrm{CDCl}_{3}\right): \delta 24.6,118.2,123.1,123.9,124.3,127.7,128.3,137.2,144.9,159.0$; MS (EI) m/z (\%): $214\left(\mathrm{M}^{+}, 100\right), 184$ (15), 168 (90), 156 (12), 141 (9), 115 (9); HRMS (EI): m/z calcd. for $\mathrm{C}_{12} \mathrm{H}_{10} \mathrm{~N}_{2} \mathrm{O}_{2} 214.0744$, found 214.0742.

\section{ACKNOWLEDGEMENTS}

This works was supported by a Grand-in-Aid for Science Research on Priority Areas (No. 18064001, Synergy of Elements) from Ministry of Education, Culture, Sports, Science and Technology, Japan.

\section{REFERENCES}

1. (a) S. P. Stanforth, Tetrahedron, 1998, 54, 263; (b) E. J. -G. Anctil and V. Snieckus, 'Metal-Catalyzed Cross-Coupling Reactions, Second, Completely Revised and Enlarged Edition, ed. by A. de Meijere and F. Diederich, 'Wiley-VCH, 2005, p. 761; (c) L. -C. Campeau and K. Fagnou, 
Chem. Soc. Rev., 2007, 36, 1058; (d) J. -P. Corbet and G. Mignani, Chem. Rev., 2009, 106, 2651.

2. For reviews, see: (a) N. Miyaura and A. Suzuki, Chem. Rev., 1995, 95, 2457; (b) A. Suzuki, 'Metal-Catalyzed Cross-Coupling Reaction ed. by F. Diederich and P. J. Stang, 'Wiley-VCH, Weinheim, 1998, p. 49; (c) N. Miyaura, 'Advances in Metal-Organic Chemistry, ed. by L. S. Liebeskind, 'JAI Press, Stamford, 1998, Vol. 6, p. 187; (d) N. Miyaura, 'Topics in Current Chemistry, 2002, 219, p. 11; (e) A. Suzuki and H. C. Brown, 'Organic Synthesis via Boranes Vol. 3 Suzuki Coupling, 'Aldrich Chemical Co., Milwaukee, 2003; (f) N. Miyaura, 'Metal-Catalyzed Cross-Coupling Reactions, Second, Completely Revised and Enlarged Edition, ed. by A. de Meijere and F. Diederich, 'Wiley-VCH, 2005, p. 41.

3. (a) H. C. Brown and M. Zaidlewicz, 'Organic Synthesis via Boranes, Vol. 2, Aldrich Chemical Co., Milwaukee, 2001; (b) D. E. Kaufmann and D. S. Matteson, 'Science of Synthesis, Houben-Weyl Methods of Molecular Transformations, Vol. 6, Thieme, Stuttgart, 2005; (c) D. Hall, 'Boronic Acids, Wiley-VCH, 2005; (d) N. Miyaura and Y. Yamamoto, 'Comprehensive Organometallic Chemistry III, Vol. 9, eds. by R. H. Crabtree, M. P. Mingos, and P. Knochel, Elsevier, Oxford, 2007, p. 145.

4. (a) S. Darses and J. -P. Genet, Eur. J. Org. Chem., 2003, 4313; (b) H. A. Stefani, R. Cella, and A. S. Vieira, Tetrahedron, 2007, 63, 3623; (c) G. A. Molander and N. Ellis, Acc. Chem. Res., 2007, 40, 275; (d) S. Derses and J. -P. Genet, Chem. Rev., 2008, 108, 288.

5. A. N. Camidge, V. H. M. Goddard, H. Gopee, N. L. Harrison, D. L. Hughes, C. J. Schubert, B. M. Sutton, G. L. Watts, and A. J. Witehead, Org. Lett., 2006, 8, 4071.

6. K. L. Billingsley and S. L. Buchwald, Angew. Chem. Int. Ed., 2008, 47, 4695.

7. (a) J. A. Soderquist, K. Matos, and A. Rane, Tetrahedron Lett., 1995, 36, 2401; (b) A. Fürstner and G. Seidel, Tetrahedron, 1995, 51, 11165.

8. C. H. Oh and S. H. Jung, Tetrahedron Lett., 2000, 41, 8513.

9. (a) P. G. Ciattini, E. Morera, and G. Ortar, Tetrahedron Lett., 1992, 33, 4815; (b) N. A. Bumagin and V. V. Bykov, Tetrahedron, 1997, 53, 14437.

10. Y. Yamamoto, M. Takizawa, X. -Q. Yu, and N. Miyaura, Angew. Chem. Int. Ed., 2008, 47, 928.

11. X. -Q. Yu, Y. Yamamoto, and N. Miyaura, Chem. Asian J., 2008, 3, 1517.

12. X. -Q. Yu, Y. Yamamoto, and N. Miyaura, Synlett, 2009, 994.

13. E. Tyrrell and P. Brookes, Synthesis, 2003, 469.

14. (a) M. Ishikura T. Mano, I. Oda, and M. Terashima, Heterocycles, 1984, 22, 2417; (b) T Murafuji, R. Mouri, Y. Sugihara, K. Takakura, Y. Mikata, and S. Yano, Tetrahedron, 1996, 52, 13933; (c) A. Bouillon, J.-C. Lancelot, J. Sopkova de Oliveira Santos, V. Collot, P. R. Bovy, and S. Rault, Tetrahedron, 2003, 59, 10043; (d) P. B. Hodgson and F. H. Salingue, Tetrahedron Lett., 2004, 45, 685; (e) P. Gros, A. Doudouh, and Y. Fort, Tetrahedron Lett., 2004, 45, 6239; (f) N. A. Jones, J. W. 
Antoon, A. L. Bowie, Jr., J. B. Borak, and E. P. Stevens, J. Heterocycl. Chem., 2007, 44, 363; (g) J. Z. Deng, D. V. Paone, A. T. Ginnetti, H. Kurihara, S. D. Dreher, S. A. Weissman, S. R. Stauffer, and C. S. Burgey, Org. Lett., 2009, 11, 345; (h) D. S. Yang, S. L. Colletti, K. Wu, M. Song, G. Y. Li, and H. C. Shen, Org. Lett., 2009, 11, 381.

15. K. Osakada, H. Onodera, and Y. Nishihara, Organometallics 2005, 24, 190 and references therein.

16. C. L. Cioffi, W. T. Spencer, J. J. Richards, and R. J. Herr, J. Org. Chem., 2004, 69, 2210.

17. S. Kotha, D. Kashinath, K. Lahiri, and R. B. Sunoj, Eur. J. Org. Chem., 2004, 4003.

18. T. Thiemann, K. Umeno, J. wang, Y. Tabuchi, K. Arima, M. Watanabe, Y. Tanaka, H. Gorohmaru, and S. Mataka, J. Chem. Soc., Perkin Trans. 1, 2002, 2090.

19. W. M. Seganish and P. DeShong, J. Org. Chem., 2004, 69, 1137.

20. A. S. B. Prasad, T. M. Stevenson, J. R. Citineni, V. Nyzam, and P. Knochel, Tetrahedron, 1997, 53, 7237.

21. T. Nokami, Y. Tomida, T. Kamei, K. Itami, and J. -i. Yoshida, Org. Lett., 2006, 8, 729.

22. A. V. Coelho, A. L. F. de Souza, P. G. De Lima, J. L. Wardell, and O. A. C. Antunes, Tetrahedron Lett., 2007, 48, 7671.

23. B. Heller, B. Sundermann, H. Buschmann, H.-J. Drexler, J. You, U. Holzgrabe, E. Heller, and G. Oehme, J. Org. Chem., 2002, 67, 4414.

24. Y. Lu, E. Plocher, and Q.-S. Hu, Adv. Synth. Catal., 2006, 348, 841. 